\title{
Phage-Based Profiling of \\ Rare Single Cells Using Nanoparticle-Directed Capture
}

Yuan Ma ${ }^{\text {ae }}$, Kangfu Chen ${ }^{\text {ae }}$,Fan Xia ${ }^{\text {ae }}$, Randy Atwal ${ }^{\mathrm{a}}$, Hansen Wanga ${ }^{\mathrm{a}}$, Sharif U. Ahmed ${ }^{\mathrm{a}}$, Lia

Cardarelli $^{\mathrm{b}}$, Irene Lui ${ }^{\mathrm{c}}$, Bill Duong ${ }^{\mathrm{a}}$, Zongjie Wang ${ }^{\mathrm{a}}$, James A. Wells ${ }^{\mathrm{d}}$, Sachdev S. Sidhu ${ }^{\mathrm{b}}$ and Shana O. Kelleyac*

${ }^{a}$ Leslie Dan Faculty of Pharmacy, University of Toronto, Toronto, Ontario, Canada.

' Terrence Donnelly Centre for Cellular and Biomolecular Research, University of Toronto, Toronto, Ontario, Canada

${ }^{\mathrm{c}}$ Department of Biochemistry, University of Toronto, Toronto, Ontario, Canada.

${ }^{\mathrm{d}}$ Department of Pharmaceutical Chemistry, University of California, San Francisco, CA 94143

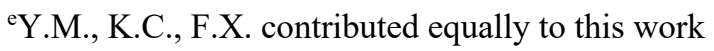

Email: shana.kelley@utoronto.ca 


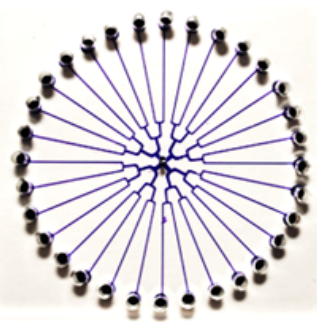

C

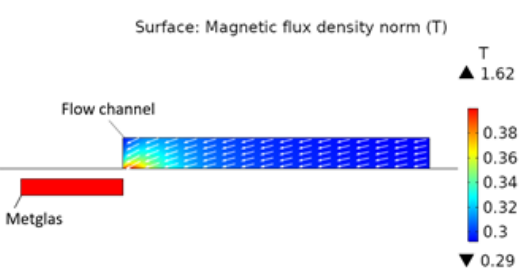

B
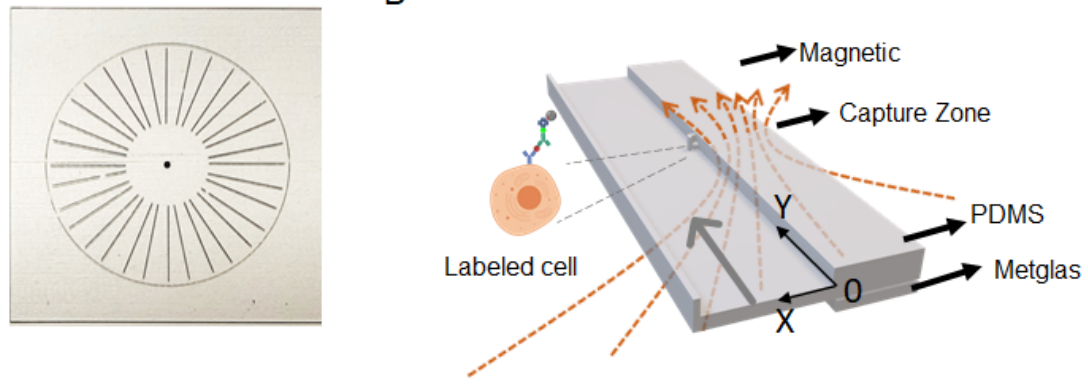

$\mathrm{D}$

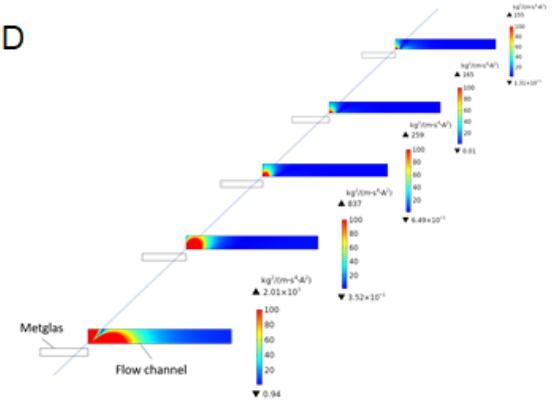

E

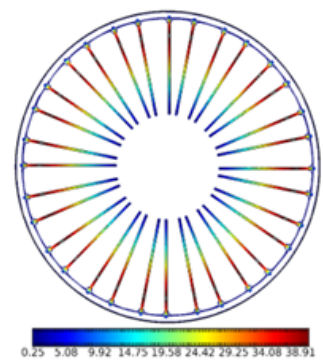

Figure S1. Design and simulation of SCP-Chip. (A) Snapshot of the SCP-Chip top and middle layer.

(B) Capture mechanism of the SCP-Chip. (C) The magnetic field was largely enhanced, and the strong magnetic gradient is generated near the magnetic guide. (D) Simulation of the magnetic field in different sections along the flow channel. (E)Magnetic flux density distribution evaluation in the whole SCPChip in the 3D model. 


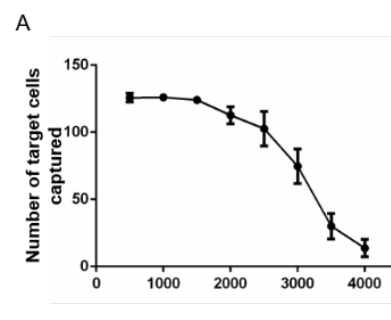

Flow rate $(\mu \mathrm{L} / \mathrm{h})$

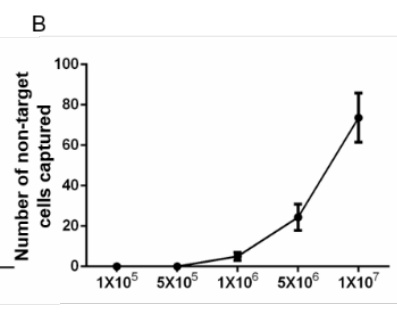

Cell concentration $(/ \mathrm{mL})$
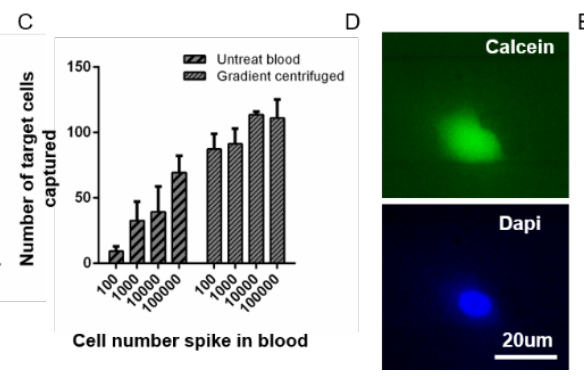

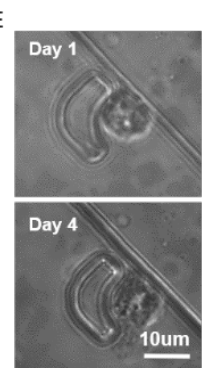

Figure S2. Optimization of parameters for the SCP-Chip. (A) The cell trap efficiency was tested at different flow rates. (B) The number of false trapped cells was tested at different cell concentrations. (C) The number of captured single cells captured using SCP-Chip at varying H460-myc cells spike in blood: Untreated blood (left); Gradient centrifuged (right). (D) Representative images of cultured trapped cells on chip. (E) Representative images of cultured CTC proliferation after $4 \mathrm{~d}$ culture. 


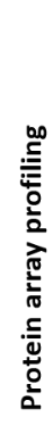

A
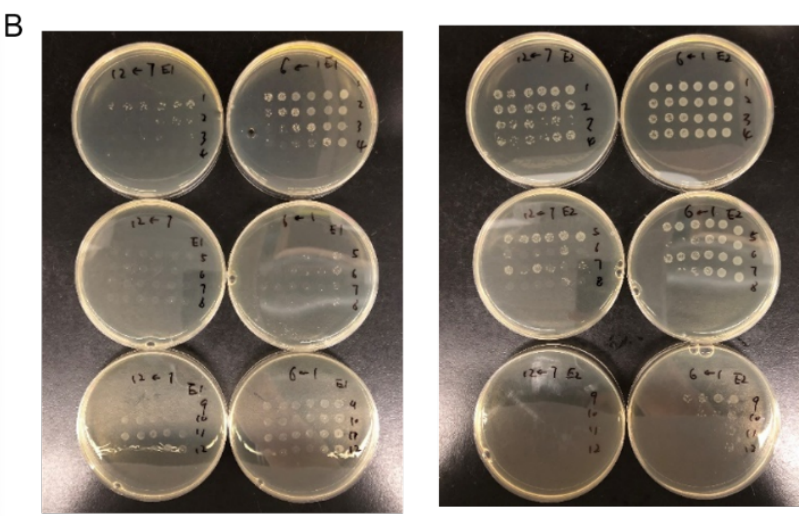

Figure S3. CRISPR EGFR KO and titration results. (A) Validation of EGFR KO by protein array profiling. (B)Titration results of microphage binding linearity test. 

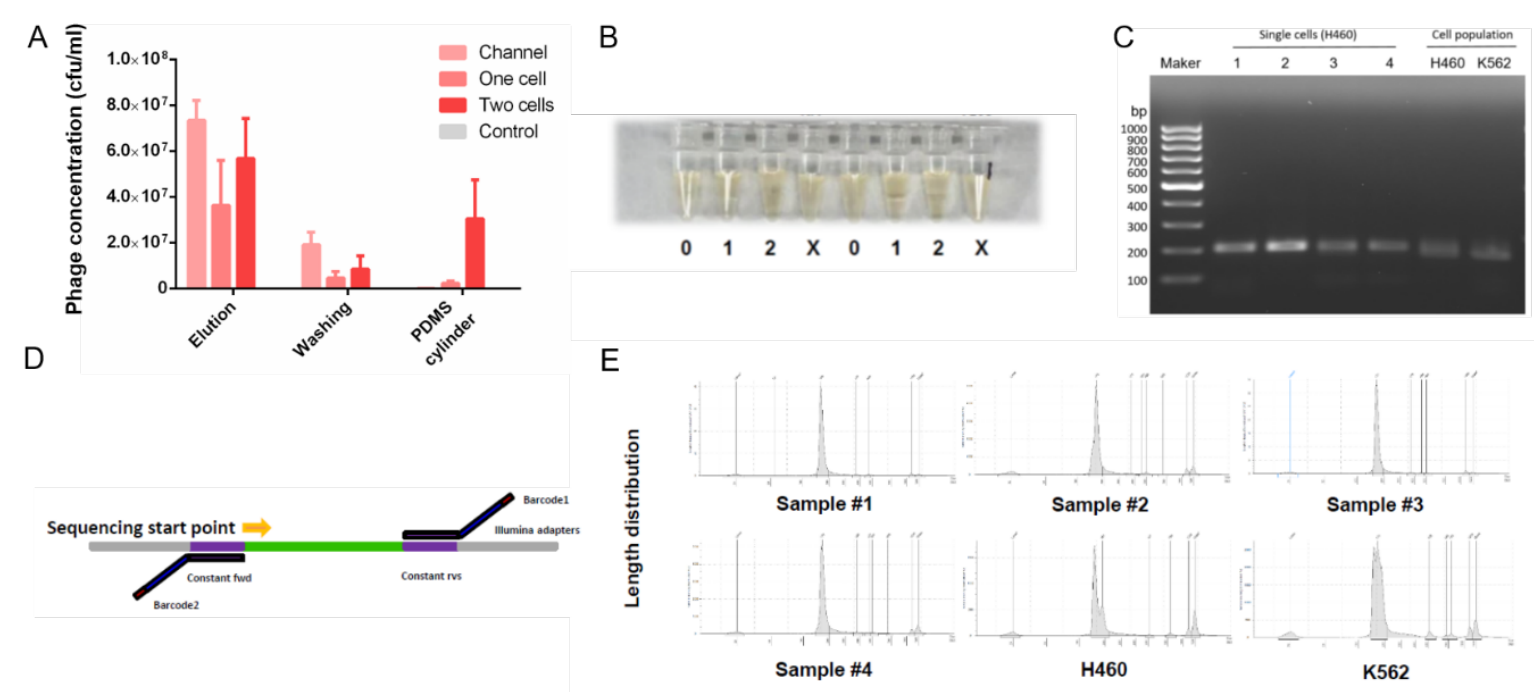

Figure S4. Optimization and preparation for the cell-bound phage propagation and NGS. (A) Comparison of the amount of the amplified phages via three methods: i. elution of phage using acidic conditions (left); ii. release of the captured cell and propagation of phage in culture (middle); punch out the PDMS cylinder containing the captured cell (right). (B) Representative images showed culturing cell-bound phage inside one or two PDMS cylinders. (C) PCR results of the genomic extraction from each sample. (D) Mechanism of the sequencing primer and barcoded design using Hiseq4000 (Illumina). (E) Comparison of sequencing length distribution of the existing $\mathrm{H} 3$ barcodes included between different samples. 

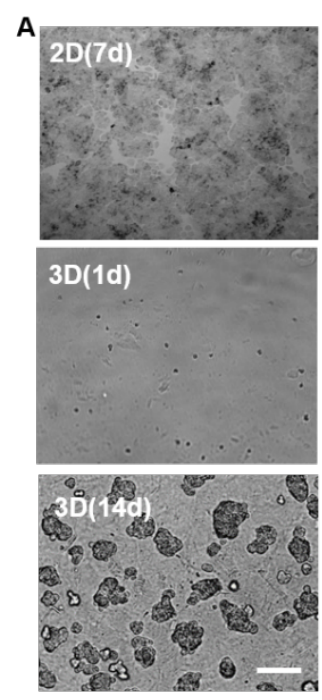

B

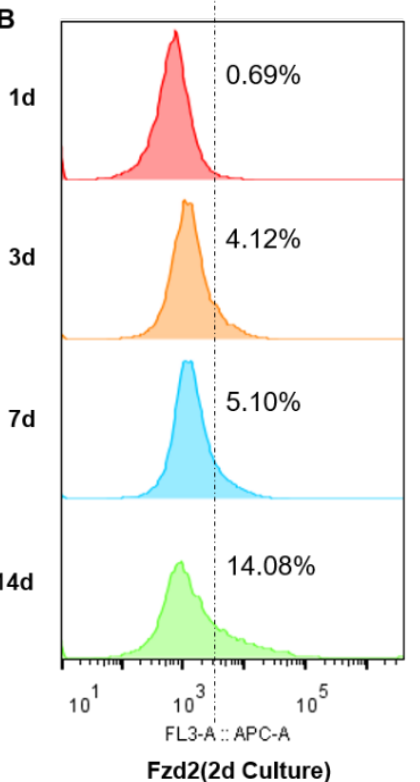

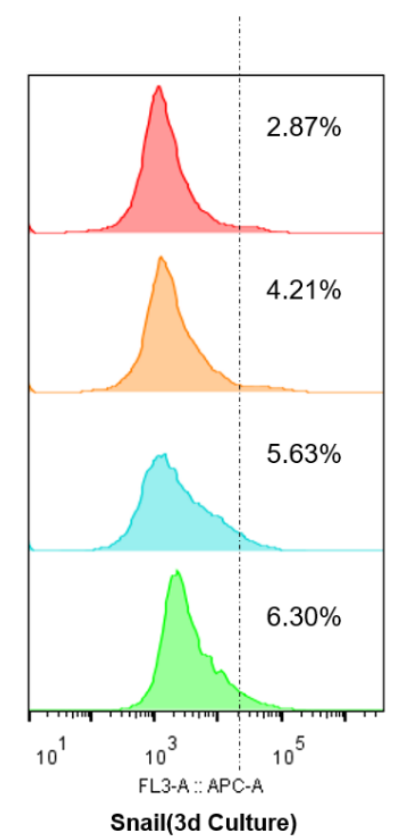

Figure S5. FZD2 expression varies across 2D and 3D culture. (A) Representative bright-field images of the H460 cells cultured as 2D monolayers (7 days) and 3D spheroids (1 day and 14 days). Scale bar is $100 \mu \mathrm{m}$. (B) FZD2 and Snail expression for 2D monolayers and 3D spheroids as a function of culture time. 


\begin{tabular}{|c|l|c|}
\hline $\begin{array}{c}\text { Parame } \\
\text { ter }\end{array}$ & \multicolumn{1}{|c|}{ Description } & Value \\
\hline$\eta$ & Dynamic viscosity of the medium & $0.001_{P a} \times{ }_{s}$ \\
\hline$\Delta_{\chi_{\text {bead }}}$ & $\begin{array}{l}\text { Relative volumetric magnetic } \\
\text { susceptibility of the nanobeads }\end{array}$ & $1.4 \times 10^{-3}$ \\
\hline$\mu_{\mathbf{0}}$ & Permeability of free space & $\begin{array}{c}4 \pi \times 10^{-7} \\
\mathrm{H} / \mathrm{m}\end{array}$ \\
\hline $\boldsymbol{R}$ & Tumor cell radius & $10 \mu \mathrm{m}$ \\
\hline $\mathbf{n}$ & $\begin{array}{l}\text { Number of nanobeads on the tumor } \\
\text { cell }\end{array}$ & $10^{5}$ \\
\hline $\boldsymbol{r}$ & Nanobead radius & $60 \mathrm{~nm}$ \\
\hline
\end{tabular}

Table 1. Parameters for COMSOL simulation.

File: The sequencing data that support the findings of this study are available in the Gene Expression Omnibus (GEO), https://www.ncbi.nlm.nih.gov/geo 